\title{
Light scattering from three-level systems: The $T$ matrix of a point dipole with gain
}

\author{
Tom Savels, * Allard P. Mosk, and Ad Lagendijk \\ Complex Photonic Systems, Department of Science and Technology, University of Twente, \\ P.O. Box 217, 7500 AE Enschede, The Netherlands
}

(Received 22 October 2004; published 18 April 2005)

\begin{abstract}
We present an extension of the $T$-matrix approach to scattering of light by a three-level system, using a description based on a Master equation. More particularly, we apply our formalism to calculate the $T$ matrix of a pumped three-level atom, providing an exact and analytical expression describing the influence of a pump on the light-scattering properties of an atomic three-level system.
\end{abstract}

DOI: 10.1103/PhysRevA.71.043814

PACS number(s): 42.50.Ct, 32.80.-t, 42.50.Gy

\section{INTRODUCTION}

In the description of the interaction of atomic ensembles with light, the atoms are often treated in the electric dipole approximation, and are therefore effectively considered to be point dipoles. The advantages of the point-dipole formalism are twofold. First, the $\delta$-function potential associated with point scatterers allows for significant mathematical simplifications compared to finite-size scatterers [1]. Second, the point-scatterer formalism allows for a transparent description of many multiple-scattering phenomena, mimicking most of the associated relevant physics [2]. The light scattering properties of point dipoles can be expressed by means of their $T$ matrix $\overleftrightarrow{T}$ which is related to the dynamic polarizability $\overleftrightarrow{\alpha}$ by $\overleftrightarrow{T}\left(\omega, \boldsymbol{r}^{\prime}, \boldsymbol{r}\right)=-(\omega / c)^{2} \overleftrightarrow{\alpha}(\omega) \delta(\boldsymbol{r}) \delta\left(\boldsymbol{r}-\boldsymbol{r}^{\prime}\right)$. If one describes the internal structure of the atoms as an effective two-level system [3] or a damped harmonic oscillator, one finds the wellknown Lorentzian expression for the linear dynamic polarizability [4] for frequencies near the resonance frequency.

In this paper, we present an extension of the point-dipole $T$ matrix to three-level atoms. Three-level atoms have been studied a lot in the past, especially in the context of lasing without inversion $[5,6]$. However, in this paper, we are focusing on a different application of three-level atoms. Our aim is to develop a transparent formulation of a point dipole with gain, based on clear physical grounds. The introduction of a pump in the point-dipole model is highly attractive because it allows, within the framework of the $T$ matrix formalism, for a description of optically amplifying atomic systems [7-10]. The results presented in this paper are a first step towards a better qualitative and quantitative understanding of the presence of gain in multiple light-scattering systems, such as atomic lattices [11] or coherent backscattering experiments [12].

Our paper is organized as follows. In Sec. II, the Master equation for an optically pumped three-level system is solved. The evolution of all atomic populations and internal coherences can then be deduced. In particular, we will focus on the system's steady-state regime. In Sec. III, we will connect the reduced density matrix of the system with its dynamic polarizability, which will lead us to the key result of

\footnotetext{
*Electronic address: t.savels@utwente.nl
}

this paper: the $T$ matrix of a point-dipole with gain. In Sec. IV, finally, we will discuss the physical meaning of our result, and elaborate on the dispersion and dissipation of a dipole in the presence of gain.

\section{MASTER EQUATION OF A PUMPED THREE-LEVEL SYSTEM}

We start by considering a three-level system $A$, shown in Fig. 1. The experimental situation we have in mind is, e.g., a hydrogenlike fine-structureless atom in a magnetic field. The $c \rightarrow a$ transition then corresponds to an allowed $\pi$ transition, whereas level $b$ is a higher-lying quickly decaying level. Decay between the energy levels $a, b$, and $c$ can occur according to the decay rates $\Gamma_{b c}$ and $\Gamma_{c a}$. The $b \rightarrow c$ transition either has a nonradiative nature, or its transition frequency is far detuned compared to the other relevant frequencies in the system. In this paper, we are interested in the simplest scheme possible that allows for incoherent pumping. Therefore, we will make the following simplifying assumptions. We assume that spontaneous emission from level $b$ to level $a$ can be neglected compared to other decay processes, hence $\Gamma_{b a} \approx 0$. Furthermore, the lifetime $\Gamma_{b c}^{-1}$ of level $b$ is chosen to be small compared to the lifetime $\Gamma_{c a}^{-1}$. Both previous restrictions on the decay rates do not affect the final results of this paper in any qualitative way, and are only introduced for mathematical simplicity. Of course, quantitative differences will arise if extra coherences imposed by the pump are taken into account through a finite $\Gamma_{b a}$, but these effects are outside the scope of this paper. Two incident electromagnetic fields $\boldsymbol{\varepsilon}_{e}$ and $\boldsymbol{\varepsilon}_{p}$ interact with $A$. The field $\boldsymbol{\varepsilon}_{p}$ serves as a pump to establish population inversion in the two-level system $a c$.

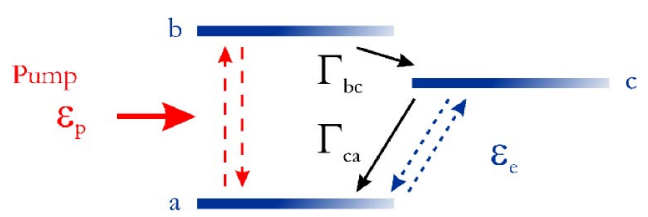

FIG. 1. (Color online) The three-level system $A$. Decay from $b$ to $c$ and from $c$ to $a$ can occur according to the decay rates $\Gamma_{b c}$ and $\Gamma_{c a}$, respectively. Decay from $b$ to $a$ is neglected. The left dashed arrows express the interaction with the pump. The right dashed arrows depict the interaction with the external probe field. 
The external probe field $\boldsymbol{\varepsilon}_{e}$ is present in order to calculate the dynamic polarizability of the $a c$ system.

We are interested in the steady-state behavior of the system $A$. We will now derive the Master equation of the system, using the standard procedure presented in [13]. The total Hamiltonian of the system $A$, the electromagnetic fields, and interactions can be written in the electric dipole and the rotating-wave approximation as

$$
\hat{H} \equiv \hat{H}_{A}+\hat{H}_{P}+\hat{H}_{R}+\hat{V}_{A R}+\hat{V}_{A E}+\hat{V}_{A P} .
$$

The atomic Hamiltonian $\hat{H}_{A}$ is given by $\hat{H}_{a b}+\hat{H}_{c}$, with

$$
\hat{H}_{a b} \equiv \hbar \omega_{1} \hat{S}_{a b}^{+} \hat{S}_{a b}^{-}, \quad \hat{H}_{c} \equiv \hbar \omega_{2} \hat{S}_{a c}^{+} \hat{S}_{a c}^{-},
$$

with $\hat{S}_{i j}^{+}, \hat{S}_{i j}^{-}$the $i j$ dipole raising and lowering operators. The Hamiltonian of the pump is given by

$$
\hat{H}_{P} \equiv \hbar \omega_{p}\left(\hat{a}_{p} \hat{a}_{p}^{\dagger}+\frac{1}{2}\right),
$$

where the operators $\hat{a}_{p}$ and $\hat{a}_{p}^{\dagger}$, respectively, annihilate and create a pump photon with frequency $\omega_{p}$. The system $A$ is coupled via the interaction $\hat{V}_{A R}$,

$$
\hat{V}_{A R} \equiv-i \hat{\boldsymbol{d}} \cdot \sum_{k \lambda}\left(\frac{\hbar \omega_{k \lambda}}{2 \varepsilon_{0} V}\right)^{1 / 2}\left(\hat{a}_{k \lambda}-\hat{a}_{k \lambda}^{\dagger}\right),
$$

to the three-dimensional multimode electromagnetic field with Hamiltonian

$$
\hat{H}_{R} \equiv \sum_{k \lambda} \hbar \omega_{k \lambda}\left(\hat{a}_{k \lambda} \hat{a}_{k \lambda}^{\dagger}+\frac{1}{2}\right)
$$

The dipole operator is denoted as $\hat{\boldsymbol{d}}$, the operators $\hat{a}$ and $\hat{a}^{\dagger}$ are the photon annihilation and creation operators, and $V$ $=L^{3}$ is the quantization volume. The external field $\boldsymbol{\varepsilon}_{e}$ has a frequency $\omega$, and interacts with the system $A$ by

$$
\hat{V}_{A E} \equiv \frac{1}{2} \hbar \Omega_{e}\left(\hat{S}_{a c}^{+} e^{-i \omega t}+\hat{S}_{a c}^{-} e^{i \omega t}\right),
$$

where the interaction strength is given by the Rabi frequency $\Omega_{e} \equiv-\boldsymbol{d}_{a c} \cdot \boldsymbol{\varepsilon}_{e} / \hbar$, with $\boldsymbol{d}_{a c}$ the $a c$ transition dipole moment.

The interaction Hamiltonian $\hat{V}_{A P}$ finally denotes the coupling of the atom to the pump,

$$
\hat{V}_{A P} \equiv g\left(\hat{S}_{a b}^{+}+\hat{S}_{a b}^{-}\right)\left(\hat{a}_{p}+\hat{a}_{p}^{\dagger}\right),
$$

with $g$ expressing the coupling strength between the pump and the system [13]. The pump field shifts the energies of the levels $a$ and $b$. This shift, which can be significant for large pump intensities, can be taken into account (see, e.g., [14]) by considering the "dressed states" or eigenstates of the Hamiltonian $\hat{H}_{D} \equiv \hat{H}_{a b}+\hat{H}_{P}+\hat{V}_{A P}$, given by

$$
\begin{aligned}
& |1(N)\rangle \equiv \sin (\theta)|a, N+1\rangle+\cos (\theta)|b, N\rangle, \\
& |2(N)\rangle \equiv \cos (\theta)|a, N+1\rangle-\sin (\theta)|b, N\rangle,
\end{aligned}
$$

graphically represented in Fig. 2. Since the energy of level $c$ is unaffected by the pump, we use the notation $|c(N)\rangle$ $\equiv|c, N\rangle$. The number of pump photons is then given by $N$, and

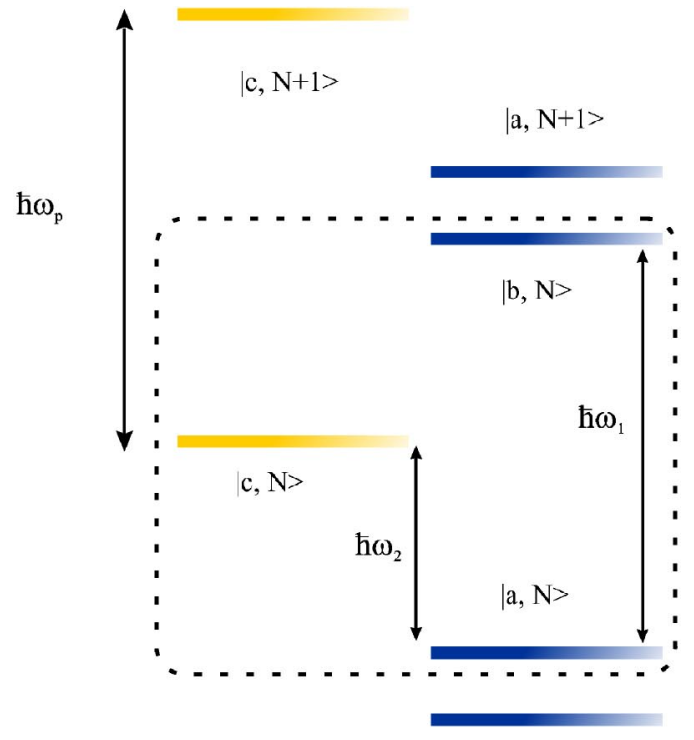

(a)

$\mid \mathrm{b}, \mathrm{N}-1>$

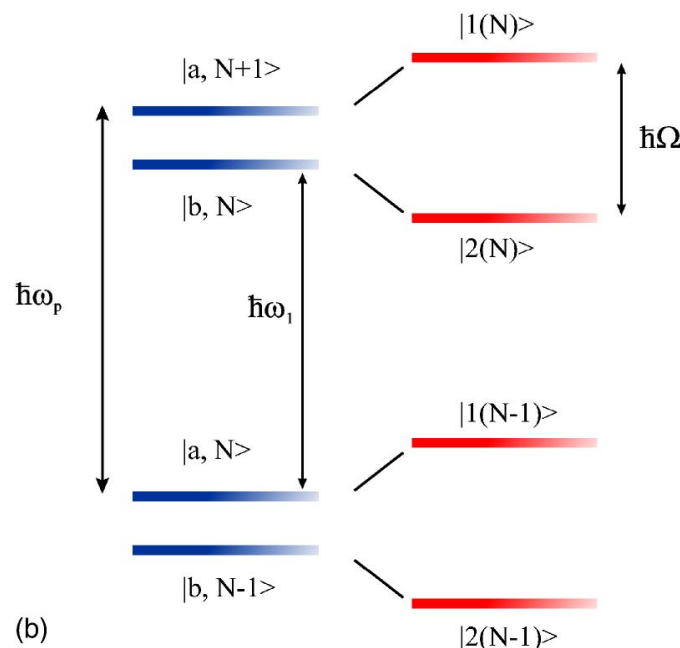

FIG. 2. (Color online) In (a), levels $|a, N\rangle,|b, N\rangle$, and $|c, N\rangle$ are shown for a different number of pump photons $N$. The energy differences between energy levels are also indicated. In (b), the dressed states $|1(N)\rangle$ and $|2(N)\rangle$ are schematically shown. The energy difference between both states is given by $\hbar \Omega=\hbar \sqrt{\delta_{p}^{2}+\Omega_{p}^{2}}$, which can easily be deduced from definitions (8).

$$
\tan 2 \theta \equiv-\frac{\Omega_{p}}{\delta_{p}}, \quad 0 \leqslant 2 \theta<\pi,
$$

where we used

$$
\hbar \Omega_{p} \equiv-\boldsymbol{d}_{a b} \cdot \boldsymbol{\varepsilon}_{p}, \quad \delta_{p} \equiv \omega_{p}-\omega_{1},
$$

with $\boldsymbol{d}_{a b}$ the $a b$ transition dipole moment. We assume for now that the distribution of pump photons is relatively narrow around a large average value $(\langle N\rangle \gg \Delta N \gg 1)$, for which $2 g \sqrt{\langle N\rangle}=\hbar \Omega_{p}$ holds (see, e.g., [13]).

The dynamics of the system $A$ can be expressed in terms of the Master equation for the reduced density matrix $\hat{\sigma}$. The Master equation is in the usual Born-Markov approximation, written in the Lindblad form, given by [15-17] 


$$
\frac{d}{d t} \hat{\sigma} \equiv \hat{\mathcal{L}} \hat{\sigma} \equiv \hat{\mathcal{L}}_{n d} \hat{\sigma}+\hat{\mathcal{L}}_{d} \hat{\sigma}
$$

The nondissipative part of the Lindblad operator can be written as

$$
\hat{\mathcal{L}}_{n d} \hat{\sigma} \equiv-\frac{i}{\hbar}\left[\hat{H}_{D}+\hat{H}_{c}+\hat{V}_{A E}, \hat{\sigma}\right],
$$

while the dissipative part is given by

$$
\begin{aligned}
\hat{\mathcal{L}}_{d} \hat{\sigma} \equiv & -\frac{\Gamma_{c a}}{2}\left(\hat{S}_{a c}^{+} \hat{S}_{a c}^{-} \hat{\sigma}+\hat{\sigma} \hat{S}_{a c}^{+} \hat{S}_{a c}^{-}\right)+\Gamma_{c a} \hat{S}_{a c}^{-} \hat{\sigma} \hat{S}_{a c}^{+}-\frac{\Gamma_{b c}}{2}\left(\hat{S}_{b c}^{+} \hat{S}_{b c}^{-} \hat{\sigma}\right. \\
& \left.+\hat{\sigma} \hat{S}_{b c}^{+} \hat{S}_{b c}^{-}\right)+\Gamma_{b c} \hat{S}_{b c}^{-} \hat{\sigma} \hat{S}_{b c}^{+} .
\end{aligned}
$$

The explicit evolution equations for all reduced densitymatrix elements are found by expanding Eq. (11) in the basis $\left\{|1(N)\rangle,\left|2\left(N^{\prime}\right)\right\rangle,\left|c, N^{\prime \prime}\right\rangle\right\}$, where $N, N^{\prime}$, and $N^{\prime \prime}$ are not necessarily equal. However, we will show in the next section that only an expansion in the basis $\{|1(N)\rangle,|2(N)\rangle,|c, N+1\rangle\}$ will be required to determine the system's $T$ matrix, and we will therefore restrict ourselves to this particular basis. If we introduce the following notations for typographical simplicity:

$$
\begin{gathered}
\sigma_{i, j}^{N, M} \equiv\langle i(N)|\hat{\sigma}| j(M)\rangle, \\
E_{i}^{N} \equiv\left\langle i(N)\left|\hat{H}_{D}+\hat{H}_{c}\right| i(N)\right\rangle, \quad i, j \in\{1,2, c\},
\end{gathered}
$$

we can focus on the evolution equations obtained by an expansion of Eq. (11) First, the evolution of the population of the dressed state $|1(N)\rangle$ is given by

$$
\begin{aligned}
\dot{\sigma}_{1,1}^{N, N}= & -i \frac{\Omega_{e}}{2} \sin (\theta)\left(e^{i \omega t} \sigma_{c, 1}^{N+1, N}-e^{-i \omega t} \sigma_{1, c}^{N, N+1}\right) \\
& +\Gamma_{c a} \sin ^{2}(\theta) \sigma_{c, c}^{N+1, N+1}-\Gamma_{b c} \cos ^{2}(\theta) \sigma_{1,1}^{N, N} \\
& +\frac{\Gamma_{b c}}{2} \sin (\theta) \cos (\theta)\left(\sigma_{1,2}^{N, N}+\sigma_{2,1}^{N, N}\right),
\end{aligned}
$$

with similar expressions for the populations $\sigma_{2,2}^{N, N}$ and $\sigma_{c, c}^{N, N}$. Secondly, coherences such as $\sigma_{1,2}^{N, N}$ evolve as

$$
\begin{aligned}
\dot{\sigma}_{1,2}^{N, N}= & -i \frac{\Omega_{e}}{2}\left(e^{i \omega t} \sin (\theta) \sigma_{c, 2}^{N+1, N}-e^{-i \omega t} \cos (\theta) \sigma_{1, c}^{N, N+1}\right) \\
& +\Gamma_{c a} \sin (\theta) \cos (\theta) \sigma_{c, c}^{N+1, N+1}-\frac{\Gamma_{b c}}{2}\left[\sigma_{1,2}^{N, N}\right. \\
& \left.-\sin (\theta) \cos (\theta) \sigma_{2,2}^{N, N}-\sin (\theta) \cos (\theta) \sigma_{1,1}^{N, N}\right]-\frac{i}{\hbar}\left(E_{1}^{N}\right. \\
& \left.-E_{2}^{N}\right) \sigma_{1,2}^{N, N},
\end{aligned}
$$

with analogous expressions for all other coherences, which are derived in the Appendix. Expanding the Master equation allows us to describe the dynamics of our system, and in particular to look at its steady-state behavior. If we examine the Master equation more closely, then two types of evolutions can be distinguished. First, the coherences are driven by the external probe field; therefore, their evolution can be separated in a quickly oscillating character (evolving typically at $\omega$ ) and a slowly decaying envelope (evolving typi- cally at $\Gamma_{c a}^{-1}$ ). Hence, a time-independent regime or "steady state" for $\hat{\sigma}(t)$ can only be obtained for the envelope of the coherences. The other matrix elements (the populations), however, do not exhibit such quick oscillatory behavior and decay to their steady-state value without any persistent oscillations. The steady-state value of the level populations $\pi_{i}$, $i \in\{a, b, c\}$, for example, is given in the small external probe field limit by

$$
\pi_{c}^{s t} \equiv \sum_{N} \sigma_{c, c}^{N, N}=\frac{\Gamma_{b c}}{\Gamma_{c a}} \pi_{b}^{s t}=\frac{W}{1+W\left(1+2 \frac{\Gamma_{c a}}{\Gamma_{b c}}\right)}=1-\pi_{b}^{s t}-\pi_{a}^{s t},
$$

where we defined the dimensionless pumping parameter

$$
W \equiv \frac{\Omega_{p}^{2}}{\Gamma_{b c} \Gamma_{c a}}, \quad 0 \leqslant W<+\infty,
$$

such that population inversion occurs in the $a c$ system for $W \geqslant 1$. Now we can describe the dynamics of the system $A$; we will focus on the connection between the steady-state solution of the Master equation and the scattering properties of the system for radiation near the $c \rightarrow a$ resonance.

\section{DERIVATION OF THE $T$ MATRIX FOR A DIPOLE WITH GAIN}

The Master equation allows us to express expectation values of atomic operators in terms of reduced density-matrix elements. In the case of a single atom, the expectation value can be interpreted as a time average due to the statistical character of the Master equation [18]. The (time) average of the operator $\hat{\boldsymbol{d}}$ associated with the $a c$ dipole transition, for example, is

$$
\langle\hat{\boldsymbol{d}}\rangle \equiv\left\langle\hat{\boldsymbol{d}}_{-}\right\rangle+\left\langle\hat{\boldsymbol{d}}_{+}\right\rangle=\boldsymbol{d}_{a c}\left[\operatorname{Tr}\left(\hat{\boldsymbol{\sigma}} \hat{S}_{a c}^{-}\right)+\operatorname{Tr}\left(\hat{\sigma} \hat{S}_{a c}^{-}\right)\right]
$$

and

$$
\begin{aligned}
\operatorname{Tr}\left(\hat{\sigma} \hat{S}_{a c}^{-}\right) \equiv & \sum_{N} \sum_{i=1,2, c}\left\langle i(N)\left|\hat{\sigma} \hat{S}_{a c}^{-}\right| i(N)\right\rangle=\sum_{N}\left[\sigma_{c, 1}^{N, N-1} \sin (\theta)\right. \\
& \left.+\sigma_{c, 2}^{N, N-1} \cos (\theta)\right],
\end{aligned}
$$

from which we can see that indeed only the reduced densitymatrix elements given by an expansion of Eq. (11) in the basis $\{|1(N)\rangle,|2(N)\rangle,|c, N+1\rangle\}$ appear in the expression for the average dipole moment. Furthermore, if we assume the pump frequency to be on resonance of the $a b$ transition, we can deduce that in steady state (defining $\omega_{2}-\omega \equiv \delta$ ),

$$
\left\langle\hat{\boldsymbol{d}}_{-}\right\rangle=-\boldsymbol{d}_{a c} e^{-i \omega t} \frac{1-W}{1+W} \frac{\Omega_{e}}{2 \delta-i \Gamma_{c a}(1+W)+\frac{2 \Omega_{e}^{2}}{2 \delta+i \Gamma_{c a}(1+W)}} .
$$

The (time) averaged atomic $a c$ dipole operator is related to the dynamic polarizability $\overleftrightarrow{\alpha}$ of the $a c$ system by (see, e.g., [4]) 


$$
\langle\hat{\boldsymbol{d}}\rangle \equiv \varepsilon_{0} \operatorname{Re}\left[\stackrel{\leftrightarrow}{\alpha}(\omega) \cdot \boldsymbol{\varepsilon}_{e} e^{-i \omega t}\right] .
$$

Both equivalent expressions (19) and (22) allow us to connect the polarizability $\overleftrightarrow{\alpha}$ with the reduced density matrix, yielding

$$
\begin{aligned}
\overleftrightarrow{\alpha}(\omega) \cdot \boldsymbol{\varepsilon}_{e}= & -\frac{1}{\hbar \varepsilon_{0}} \boldsymbol{d}_{a c}\left(\boldsymbol{d}_{a c} \cdot \boldsymbol{\varepsilon}_{e}\right) \frac{1-W}{1+W} \\
& \times \frac{1}{-\delta+i \frac{\Gamma_{c a}}{2}(1+W)-\frac{2 \Omega_{e}^{2}}{2 \delta+i \Gamma_{c a}(1-W)}},
\end{aligned}
$$

which simplifies for small external probe fields to

$$
\overleftrightarrow{\alpha}(\omega)=-\overleftrightarrow{\alpha}_{0} \frac{1-W}{1+W} \frac{1}{\omega-\omega_{2}+i \frac{\Gamma_{c a}}{2}(1+W)} .
$$

The static polarizability is given by

$$
\overleftrightarrow{\alpha}_{0} \equiv \alpha_{0} \boldsymbol{\mu} \otimes \boldsymbol{\mu}, \quad \alpha_{0} \equiv \frac{2}{\omega_{2} \hbar \varepsilon_{0}},
$$

where " $\otimes$ " denotes the tensor product of two vectors, and $\boldsymbol{\mu}$ is the unit vector parallel to $\boldsymbol{d}_{a c}$.

It is important to note that the same expression (23) is obtained if the dressing of the levels $a$ and $b$ is omitted and the optical Bloch equations are used instead of the Master equation. The incoherent pumping mechanism only appears in the polarizability as a parameter $W$, without causing any detuning effects.

The scattering properties of a point-dipole-or, more generally, any scattering object—can be expressed by its $T$ ma-

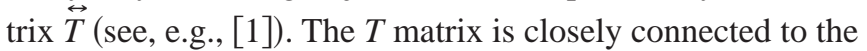
dynamic polarizability of the scatterer. For a point dipole located at $\boldsymbol{r}=\mathbf{0}$, both scattering quantities are related by

$$
\begin{aligned}
\left\langle\boldsymbol{r}|\overleftrightarrow{T}(\omega)| \boldsymbol{r}^{\prime}\right\rangle= & -\left(\frac{\omega_{2}}{c}\right)^{2} \overleftrightarrow{\alpha}(\omega) \delta(\boldsymbol{r}) \delta\left(\boldsymbol{r}-\boldsymbol{r}^{\prime}\right)=t(\omega) \boldsymbol{\mu} \otimes \boldsymbol{\mu} \delta(\boldsymbol{r}) \delta(\boldsymbol{r} \\
& \left.-\boldsymbol{r}^{\prime}\right),
\end{aligned}
$$

with a matrix element $t(\omega)$,

$$
\begin{aligned}
t(\omega) \equiv & \alpha_{0}\left(\frac{\omega_{2}}{c}\right)^{2} \frac{1-W}{1+W} \frac{1}{2} \\
& \times \frac{\omega_{2}}{\omega-\omega_{2}+i \frac{\Gamma_{c a}}{2}(1+W)-\frac{\Omega_{e}^{2}}{2 \omega_{2}-2 \omega+i \Gamma_{c a}(1+W)}},
\end{aligned}
$$

which is nonlinear in the incident probe field (through $\Omega_{e}$ ). Both $\delta$ functions appearing in Eq. (26) express the local character of the scatterer; the anisotropy of the $T$ matrix is clearly due to the preferential orientation induced by the transition dipole moment. In the absence of pumping ( $W$ $=0$ ) and for small external probe fields, we recover the expression for the linear dynamic polarizability of a two-level atom, which satisfies the optical theorem [2] expressing energy conservation,

$$
-\left.\operatorname{Im}\left[\frac{t(\omega)}{\omega_{2} / c}\right]\right|_{\substack{W=0 \\ \Omega_{e} \rightarrow 0}}=\left.\frac{|t(\omega)|^{2}}{6 \pi}\right|_{\substack{W=0 \\ \Omega_{e} \rightarrow 0}} .
$$

For the optical theorem to hold, the static polarizability must satisfy

$$
\alpha_{0}=\frac{6 \pi}{\left(\omega_{2} / c\right)^{3}} \frac{\Gamma_{c a}}{\omega_{2}},
$$

which, if substituted in Eq. (27), yields the final expression for the $T$ matrix element of a point dipole with gain for arbitrary pump and external probe field intensity. In the limit for small external probe fields, Eq. (27) then reduces to

$$
t(\omega)=\frac{3 \pi}{\omega_{2} / c} \frac{\Gamma_{c a}}{\left(\omega-\omega_{2}+i \frac{\Gamma_{c a}}{2}(1+W)\right)} \frac{1-W}{1+W} .
$$

Expressions (26) and (30) [or more generally, Eq. (27)] are the key results of this paper. In the next section, we will elaborate on their properties and physical consequences.

\section{PROPERTIES OF THE $T$ MATRIX FOR A DIPOLE WITH GAIN}

The $T$ matrix (26) fully expresses the scattering properties of a point dipole with gain and satisfies the Kramers-Kronig relations [19], which can be easily verified.

The pump influences the $T$ matrix in a clear physical way. The most obvious effect of the pump is to induce changes of the (time) averaged populations of the levels $a$ and $c$, which leads to the multiplication of the dynamic polarizability with a factor $(1-W) /(1+W)$. Remarkably, the multiplication factor becomes negative if population inversion is present in the ac system $(W>1)$, expressing the fact that not only the imaginary, but also the real part of the dynamic polarizability is drastically changed by pumping. In other words, not only does absorption change into gain, but the dispersion relation also changes, as is shown in Fig. 3. Besides changing the sign of the dynamic polarizability, the gain also effectively broadens the $a$ state by a factor $(1+W)$, which is equivalent with a decrease of the $Q$ factor of the $c \rightarrow a$ resonance.

Secondly, it is intuitively clear that the optical theorem does not hold any longer as a nonzero pump is applied. We will now show that this is indeed the case. If an external probe field with polarization vector $\boldsymbol{\epsilon}$ is incident on the point dipole we consider here, then the dipole scattering cross section $\sigma_{s c a}$ and extinction cross section $\sigma_{e x t}$ are given by

$$
\begin{gathered}
\sigma_{s c a}=+\frac{(\boldsymbol{\mu} \cdot \boldsymbol{\epsilon})^{2}}{6 \pi}|t(\omega)|^{2}, \\
\sigma_{e x t}=-\frac{(\boldsymbol{\mu} \cdot \boldsymbol{\epsilon})^{2}}{\omega / c} \operatorname{Im}[t(\omega)] .
\end{gathered}
$$

Both scattering cross sections depend [through $t(\omega)$ ] on the applied pumping intensity. The albedo of the $a c$ system is 

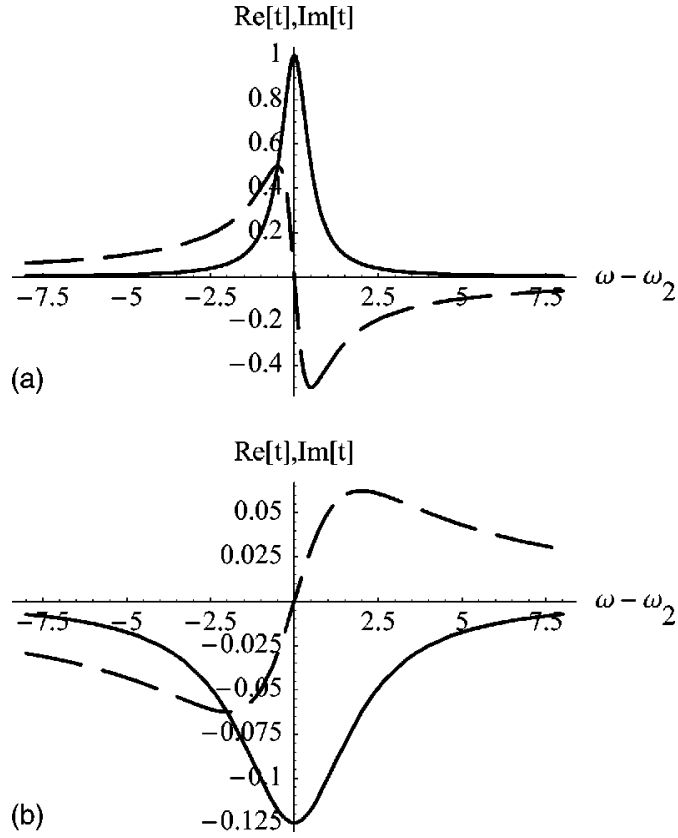

FIG. 3. The absorption (solid line), described by $\operatorname{Im}[t(\omega)]$, and the dispersion (dashed line), given by $\operatorname{Re}[t(\omega)]$ for the pumped three-level atom we consider, as a function of the detuning $\omega-\omega_{2}$ in units of $\Gamma_{c a}$ (a) without a pump $(W=0)(\mathrm{b})$ for a pumping intensity in the population inversion regime $(W=3)$. The graphs are scaled such that $\operatorname{Im}\left[t\left(\omega_{2}\right)\right]=1$ in (a).

defined as the ratio of the elastic scattering cross section and the extinction cross section, which can be written for small incident probe fields as

$$
a \equiv \frac{\sigma_{s c a}}{\sigma_{e x t}}=\frac{1-W}{(1+W)^{2}},
$$

where $(1-a)$ is the fraction of the incident probe field which is taken away from the incident beam but not transformed into scattered light. If the optical theorem is satisfied, the albedo is one, as can be seen from expression (28). Therefore, if the optical theorem does not hold any longer, we expect $a$ to be smaller than unity. To show that this is indeed true, we plotted the albedo (32) in Fig. 4 as a function of the reduced pumping parameter $W$. As soon as the dipole has internal population inversion $(W>1)$, the albedo becomes negative, which indicates that the point dipole then has a negative extinction cross section, as we expect. Obviously, the scattering cross section is-by definition-always positive and the effect of the pump is manifested purely as a decreasing of the scattered field for increasing pump. Furthermore, the extinction cross section is, in absolute value, always larger than the scattering cross section for nonzero pump. In other words, some of the incident probe field is taken away from the incident beam, but not scattered elastically. The presence of inelastic scattering is no surprise, since the total intensity emitted by the dipole is proportional to the population of the upper state $c$, whereas the coherent intensity emitted by the dipole is proportional to the amount of coherence between $a$ and $c$ (see, e.g., [13]). Both intensities are only equal (all scattering is then elastic) in the absence of

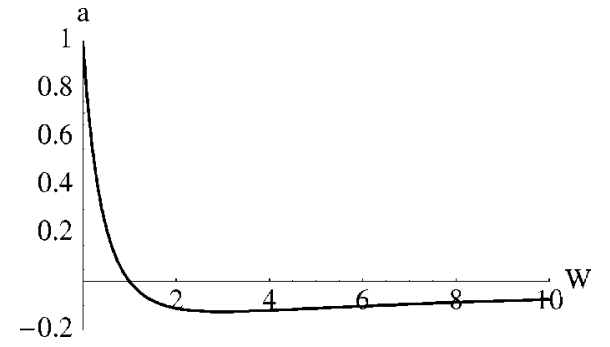

FIG. 4. The albedo $a c$ system as a function of the reduced pumping parameter $W$.

a pump and in the small external probe field limit. As soon as a pump is applied, or for larger external probe fields [20], some of the light is scattered inelastically.

Finally, we see from expressions (31) that the optical theorem is also satisfied for the nontrivial values $W=1$ and $W \rightarrow+\infty$. The latter two pumping values correspond to pumping intensities for which the $T$ matrix vanishes (therefore the optical theorem holds), but the reason why is clearly different for both cases: for $W=1$, populations are, on average, equally distributed among levels $a$ and $c$, preventing the building up of an average scattered field; for $W \rightarrow+\infty$, on the other hand, the linewidth broadening of the $c \rightarrow a$ transition induced by the pump inhibits scattering. We stress that the absence of light scattering for $W=1$ has to be interpreted in a statistical sense: the $T$ matrix is deduced from the (statistical) Master equation (11), and is therefore a time-averaged property of the system. In other words, for $W=1$, the pumped point dipole does not scatter light on average. Furthermore, the transparency in our system is caused by a fully incoherent pumping scheme, contrary to, e.g., electromagnetically induced transparency [21].

Finally, we note that the strong dependence of the albedo on the applied pumping intensity could lead to interesting experimental work on, e.g., coherent backscattering [22,23], since the width of the coherent backscattering cone is closely related to the albedo of the scatterers in the multiple scattering medium considered.

\section{SUMMARY}

The aim of this paper was to find the light-scattering properties of a pumped point dipole, modeled as a three-level system. The resulting $T$ matrix (30) [or more generally, Eq. (27)] is surprisingly intuitive. The influence of the pump can be characterized by a single dimensionless parameter $W$, expressing both a decrease of the $Q$ factor and a decrease of the $T$ matrix. Physically, the presence of gain not only causes the dipole to scatter partially inelastically, but also induces important changes in its dispersion and dissipation.

\section{ACKNOWLEDGMENTS}

We would like to thank M. Wubs and W. L. Vos for inspiring discussions. This work is part of the research program of the "Stichting voor Fundamenteel Onderzoek der Materie" (FOM), which is financially supported by the "Nederlandse Organisatie voor Wetenschappelijk Onderzoek" (NWO). 


\section{APPENDIX A: EXPANSION OF THE MASTER EQUATION}

The aim of this appendix is to show the expansion of the Master equation (11) in the basis $\{|1(N)\rangle,|2(N)\rangle,|c, N+1\rangle\}$. The reduced density-matrix elements which are diagonal in the atomic states evolve as

$$
\begin{aligned}
\dot{\sigma}_{1,1}^{N, N}= & -i \frac{\Omega_{e}}{2} \sin (\theta)\left(\sigma_{c, 1}^{N+1, N} e^{i \omega t}-\sigma_{1, c}^{N, N+1} e^{-i \omega t}\right) \\
& +\Gamma_{c a} \sin ^{2}(\theta) \sigma_{c, c}^{N+1, N+1}-\Gamma_{b c} \cos ^{2}(\theta) \sigma_{1,1}^{N, N} \\
& +\frac{\Gamma_{b c}}{2} \sin (\theta) \cos (\theta)\left(\sigma_{1,2}^{N, N}+\sigma_{1,2}^{N, N}\right), \\
\dot{\sigma}_{2,2}^{N, N}= & -i \frac{\Omega_{e}}{2} \cos (\theta)\left(\sigma_{c, 2}^{N+1, N} e^{i \omega t}-\sigma_{2, c}^{N, N+1} e^{-i \omega t}\right) \\
& +\Gamma_{c a} \cos { }^{2}(\theta) \sigma_{c, c}^{N+1, N+1}-\Gamma_{b c} \sin ^{2}(\theta) \sigma_{2,2}^{N, N} \\
& +\frac{\Gamma_{b c}}{2} \sin (\theta) \cos (\theta)\left(\sigma_{1,2}^{N, N}+\sigma_{1,2}^{N, N}\right), \\
\dot{\sigma}_{c, c}^{N+1, N+1}=- & i \frac{\Omega_{e}}{2} \sin (\theta)\left(\sigma_{1, c}^{N, N+1} e^{-i \omega t}-\sigma_{c, 1}^{N+1, N} e^{i \omega t}\right)-i \frac{\Omega_{e}}{2} \cos (\theta) \\
& \times\left(\sigma_{2, c}^{N, N+1} e^{-i \omega t}-\sigma_{c, 2}^{N+1, N} e^{i \omega t}\right)-\Gamma_{c a} \sigma_{c, c}^{N+1, N+1} \\
+ & \Gamma_{b c} \cos { }^{2}(\theta) \sigma_{1,1}^{N+1, N+1}-\Gamma_{b c} \sin (\theta) \cos (\theta)\left(\sigma_{1,2}^{N+1, N+1}\right. \\
+ & \left.\sigma_{2,1}^{N+1, N+1}\right)+\Gamma_{b c} \sin ^{2}(\theta) \sigma_{2,2}^{N+1, N+1},
\end{aligned}
$$

while the elements which are off-diagonal in the atomic states evolve as

$$
\begin{aligned}
\dot{\sigma}_{1,2}^{N, N}= & -i \frac{\Omega_{e}}{2}\left[\sin (\theta) \sigma_{c, 2}^{N+1, N} e^{i \omega t}-\cos (\theta) \sigma_{1, c}^{N, N+1} e^{-i \omega t}\right] \\
& +\Gamma_{c a} \sin (\theta) \cos (\theta) \sigma_{c, c}^{N+1, N+1}-\frac{\Gamma_{b c}}{2}\left[\sigma_{1,2}^{N, N}\right. \\
& \left.-\sin (\theta) \cos (\theta) \sigma_{2,2}^{N, N}-\sin (\theta) \cos (\theta) \sigma_{1,1}^{N, N}\right]-\frac{i}{\hbar}\left(E_{1}^{N}\right.
\end{aligned}
$$

$$
\begin{gathered}
\left.-E_{2}^{N}\right) \sigma_{1,2}^{N, N}, \\
\dot{\sigma}_{1, c}^{N, N+1} e^{-i \omega t}=-i \frac{\Omega_{e}}{2}\left[\sin (\theta) \sigma_{c, c}^{N+1, N+1}-\sin (\theta) \sigma_{1,1}^{N, N}\right. \\
\left.-\cos (\theta) \sigma_{1,2}^{N, N}\right]-\frac{\Gamma_{c a}}{2} \sigma_{1, c}^{N, N+1} \\
-\frac{\Gamma_{b c}}{2}\left[\cos ^{2}(\theta) \sigma_{1, c}^{N, N+1} e^{-i \omega t}\right. \\
\left.-\sin (\theta) \cos (\theta) \sigma_{2, c}^{N, N+1} e^{-i \omega t}\right]+\frac{i}{\hbar}\left(E_{c}^{N+1}\right. \\
\left.-E_{1}^{N}\right) \sigma_{1, c}^{N, N+1} e^{-i \omega t}, \\
\dot{\sigma}_{2, c}^{N, N+1} e^{-i \omega t}=-i \frac{\mathrm{A})}{2}\left[\cos (\theta) \sigma_{c, c}^{N+1, N+1}-\cos (\theta) \sigma_{2,2}^{N, N}\right. \\
\left.-\sin (\theta) \sigma_{1,2}^{N, N}\right]-\frac{\Gamma_{c a}}{2} \sigma_{2, c}^{N, N+1} \\
+\frac{\Gamma_{b c}}{2}\left[\sin (\theta) \cos (\theta) \sigma_{1, c}^{N, N+1} e^{-i \omega t}\right. \\
\left.\Omega_{e}(\theta) \sigma_{2, c}^{N, N+1} e^{-i \omega t}\right]+\frac{i}{\hbar}\left(E_{c}^{N+1}-E_{2}^{N}\right) \sigma_{2, c}^{N, N+1} e^{-i \omega t}
\end{gathered}
$$

and

$$
\begin{gathered}
\dot{\sigma}_{2,1}^{N, N}=\left(\dot{\sigma}_{1,2}^{N, N}\right)^{*}, \\
\dot{\sigma}_{c, 1}^{N+1, N}=\left(\dot{\sigma}_{1, c}^{N, N+1}\right)^{*}, \\
\dot{\sigma}_{c, 2}^{N+1, N}=\left(\dot{\sigma}_{2, c}^{N, N+1}\right)^{*} .
\end{gathered}
$$

[1] P. de Vries, D. V. van Coevorden, and A. Lagendijk, Rev. Mod. Phys. 70, 447 (1980).

[2] A. Lagendijk and B. A. van Tiggelen, Phys. Rep. 270, 143 (1996).

[3] L. Allen and J. H. Eberly, Optical Resonance and Two-level Atoms (Dover Publications, New York, 1987).

[4] R. Loudon, The Quantum Theory of Light, 3rd ed. (Oxford University Press, New York, 2000).

[5] K. K. Meduri et al., Quantum Opt. 6, 287 (1994).

[6] A. K. Popov, S. A. Myslivets, G. Jin-ye, Z. Han-zhuang, and B. Wellegehausen, Chin. Phys. 9, 124 (2000).

[7] J. McKeever, A. Boca, A. D. Boozer, J. R. Buck, and H. J. Kimble, Nature (London) 425, 268 (2003).

[8] K. An, J. J. Childs, R. R. Dasari, and M. S. Feld, Phys. Rev. Lett. 73, 3375 (1994).
[9] D. Meschede, H. Walther, and G. Müller, Phys. Rev. Lett. 54, 551 (1985).

[10] C. Ginzel, H.-J. Briegel, U. Martini, B.-G. Englert, and A. Schenzle, Phys. Rev. A 48, 732 (1992).

[11] M. Greiner, O. Mandel, T. Esslinger, T. W. Hnsch, and I. Bloch, Nature (London) 415, 39 (2002).

[12] T. Wellens, B. Gremaud, D. Delande, and C. Miniatura, Phys. Rev. A 70, 023817 (2004).

[13] C. Cohen-Tannoudji, J. Dupont-Roc, and G. Grynberg, AtomPhoton Interactions (Wiley, New York, 1998).

[14] H. J. Carmichael, Statistical Methods in Quantum Optics 1 (Springer-Verlag, Berlin, 2002).

[15] G. Lindblad, Commun. Math. Phys. 48, 119 (1976).

[16] A. R. P. Rau and R. A. Wendell, Phys. Rev. Lett. 89, 220405 (2002). 
[17] M. O. Scully and M. S. Zubairy, Quantum Optics (Cambridge University Press, Cambridge, 1997).

[18] L. Mandel and E. Wolf, Optical Coherence and Quantum Optics (Cambridge University Press, Cambridge, 1995).

[19] N. W. Ashcroft and N. D. Mermin, Solid State Physics (Saunders College, Philadelphia, 1976).

[20] T. Chanelière, D. Wilkowski, Y. Bidel, R. Kaiser, and C.
Miniatura, Phys. Rev. E 70, 036602 (2004).

[21] S. E. Harris, J. E. Field, and A. Imamoğlu, Phys. Rev. Lett. 64, 1107 (1990).

[22] D. V. Kupriyanov, I. M. Sokolov, P. Kulatunga, C. I. Sukenik, and M. D. Havey, Phys. Rev. A 67, 013814 (2003).

[23] M. B. van der Mark, M. P. van Albada, and Ad Lagendijk, Phys. Rev. B 37, 3575 (1988). 